\title{
C-terminal 'Tails' of Tubulin Revealed by Negative Stain EM Tomography
}

\author{
Andrea Fera ${ }^{1}$, Dan L. Sackett ${ }^{2}$ and Thomas S. Reese ${ }^{1}$ \\ 1. Laboratory of Neurobiology, National Institute of Neurological Disorders and Stroke; \\ 2. Program in Physical Biology, Eunice Kennedy Shriver National Institute of Child Health and Human \\ Development; National Institutes of Health, Bethesda, Maryland 20892, USA
}

Many proteins in the cell interact with the surface of microtubules, where the most C-terminal part of tubulin is exposed. This consists of the terminal two helices of $\alpha$ - and $\beta$-tubulin and an unstructured "tail" peptide that extends outward from the final helix of each subunit. These tails are flexible, anionic (due to high glutamic acid content), and vary in average length from 10 residues for $\alpha$-tubulin to 20 residues for $\beta$-tubulin. The major sequence differences between different isotypes of $\alpha$ and $\beta$ tubulin are located in the tails, as are most post-translational modifications of the protein, some of which are unique to tubulin. These features, conserved throughout the eukaryotic world, demonstrate the clear biological importance of these structural elements of tubulin. The tails are displayed on the surface of the microtubule, forming a charged brush with which any protein which binds to the surface of a microtubule must interact. Despite the clear functional significance of these tails, their structure is unknown, since all of the methods that have provided high resolution information about tubulin and microtubule structure rely on averaging. Since the tails vary in sequence and post-translational modifications, and since they are conformationally mobile, their structure is not revealed by these techniques. Thus there is a need for new methods or approaches that do not rely on averaging.

This work presents results obtained combining the electron microscopy tomography method with a recent method of sample preparation [1] which allows nanometer-scale resolution without averaging among many samples. Further, results have been improved using modern aberration-corrected microscopes and refined data analysis procedures.

We used stable macromolecular assemblies obtained with the antimitotic drug Cryptophycin-1 (Cr). $\mathrm{Cr}$ is a potential anti-cancer treatment which arrests cell growth by destabilizing microtubules. When added in vitro to either dimeric tubulin or microtubules, $\mathrm{Cr}$ induces the formation of single protofilament rings that are highly monodisperse, composed of eight tubulin dimers resulting in a diameter of $\sim 28 \mathrm{~nm}$ and thickness of $\sim 4 \mathrm{~nm}$ [2]. Here we apply negative stain tomography [1] to Cr-tubulin rings. This technique, based on a beam stable organo-tungsten negative stain, when combined with proper data analysis, allows construction of tomograms that reveal nanometer scale details. In particular, we focused on the C-termini predicted $[3,4]$ to protude from each tubulin monomer.

The sample has been embedded in a heavier than usual layer of stain so as to entirely embed the rings. A tungsten-based negative stain (NanoW, Nanoprobes) has been chosen [1] which, although destabilizing for microtubules, has no effect on these rings. The Cr-ring is a single protofilament, curved by $\mathrm{Cr}$ such that the external surface of the microtubule is the interior part of the ring. Experiments have been carried out with a Tecnai TF-30 microscope, but also with a monochromatized and aberration corrected TEAM I. Various data analysis methods have been compared in a systematic way (SIRT, CTF correction, WBP with IMOD, sub-tomogram alignment...). 
The structure of $\mathrm{Cr}$ tubulin rings is shown in series of en face virtual sections $\sim 4 \AA$ thick calculated using IMOD, and positioned parallel to the plane of the rings.
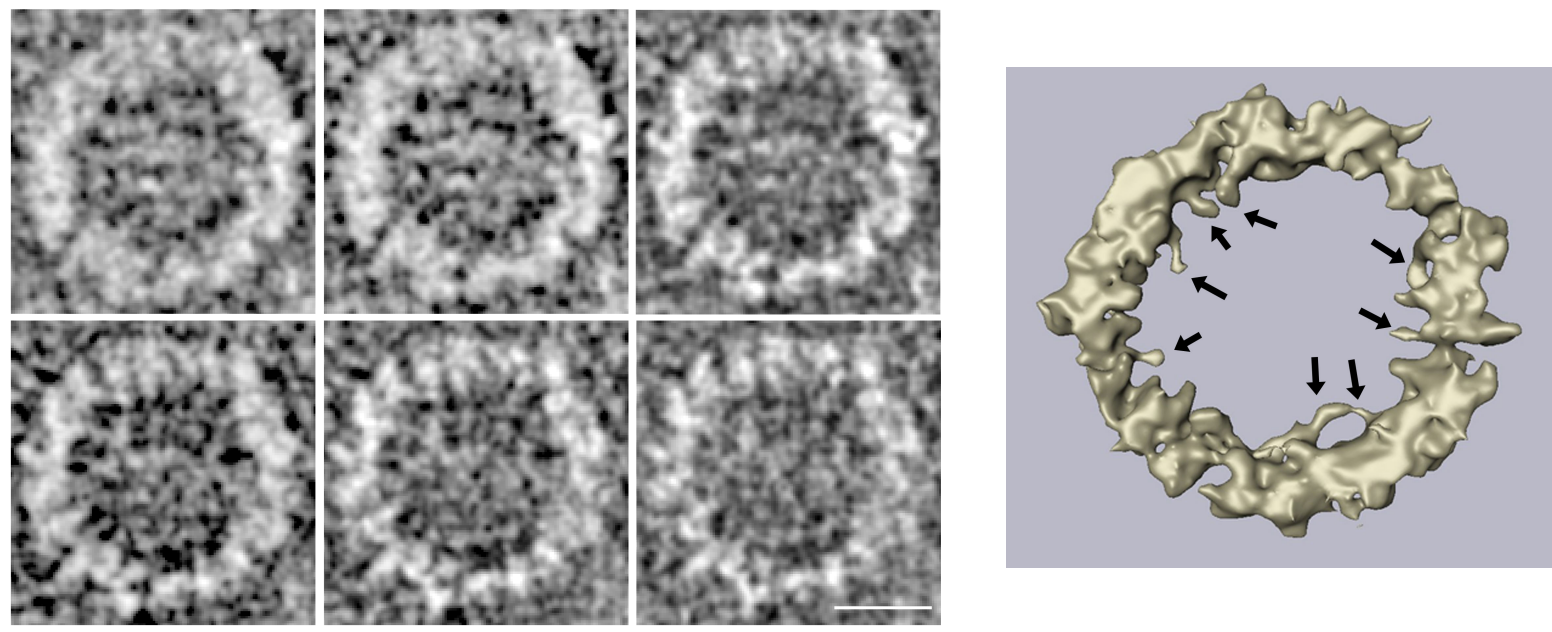

Figure 1: LEFT: En-face consecutive virtual sections (from Left to Right and Top to Bottom) of negative stained Cr-rings. RIGHT: rendering obtained imposing a constant threshold to each virtual section on the left. Black arrows on the right indicate putative C-termini, all found at the bottom of this Cr-ring. Pixel size: $0.36 \mathrm{~nm}$. Scale bar: $10 \mathrm{~nm}$.

Combining experimental optimizations increases $\mathrm{S} / \mathrm{N}$ significantly, but the difference in scattering contrast between the stain and the objects remains the most important factor. A result $\sim 50 \%$ accurate can be obtained without aberration corrections. Indeed 8 of the predicted 16 filaments of a ring can be seen in the rendering above. It is important to note that the rendering of the virtual sections on the left has been obtained by imposing a constant threshold at each virtual section. The $\mathrm{S} / \mathrm{N}$ has been improved significantly for details of dimensions comparable with the pixel size after accounting for the contrast transfer function (CTF). We have also compared different algorithm to calculate tomograms, but IMOD, which is more user friendly, seems to produce accurate results.

Tails are found regularly spaced and all at one side of the ring, as predicted. They are also found to apparently interact with the globular part of the protein, also as predicted [3]. We plan to extend our findings by tagging the extreme $\mathrm{C}$-terminal end of $\alpha$-tubulin with $1.4 \mathrm{~nm}$ nanogold using enzymatically targeted labeling [5]. Results will also be compared with tomograms measured from rings obtained using enzymatically modified tubulin lacking both C-terminal tails [4].

\section{References}

[1] A Fera, JE Farrington, J Zimmerberg and TS Reese, Microsc. and Microan. 18 (2012), p.331.

[2] NR. Watts, N Cheng, W West, AC. Steven, and DL. Sackett, Biochemistry 41 (2002), p. 12662.

[3] H Freedman, T Luchko, RF Luduena, and JA. Tuszynski, Proteins 79 (2011), p. 2968.

[4] DL Sackett and J Wolff, J Biol Chem. 261 (1986), p.9070.

[5] A Banerjee, T Panosian, K Mukherjee, R Ravinda, S Gal, DLSackett, S Bane ACS Chem Biol 5 (2010), p.777.

[6] Experiments at the NIBIB facility of the NIH campus, in Bethesda, MD and at the TEAM I microscope of NCEM-LBNL in Berkley, CA. This work was supported by a contract award from NINDS (AF), by the Intramural Research Program of NINDS (TR), and by the Intramural Research Program of NICHD (DS). 